\title{
MEDIÇÃO DE VAZÃO DO FLUÍDO DE ARREFECIMENTO COM MEDIDOR TIPO TURBINA
}

\author{
F. V. RADEL ${ }^{1}$, M. ESPOSITO ${ }^{1}$ \\ ${ }^{1}$ Universidade Federal de Pelotas, Centro de Engenharias, Engenharia de Controle e \\ Automação \\ E-mail para contato: marcelo.esposito@ufpel.edu.br
}

\begin{abstract}
RESUMO - Este trabalho apresenta um método para a medição da vazão do fluido de arrefecimento que circula na camisa de um reator, utilizando um medidor do tipo turbina. Um sensor foi acoplado a um hidrômetro convencional para a realização da aquisição de dados com o software Matlab. Os valores medidos apresentaram boa concordância com resultados experimentais obtidos a partir da interrupção da corrente de fluido e posterior medição do volume e do tempo decorrido durante o desvio do fluxo, para a determinação da vazão.
\end{abstract}

\section{INTRODUÇÃO}

Devido ao crescimento e a expansão dos processos contínuos industriais, a medição da vazão de fluidos torna-se cada vez mais necessária e importante (Bergman, 2011). Neste trabalho, vazão é a taxa de escoamento de um fluido, ou seja, o volume de fluido que passa por um determinado conduto por unidade de tempo.

O presente trabalho objetiva a determinação da vazão do fluido de arrefecimento, água, empregado usualmente em processos de polimerização. O método de medição apresentado foi desenvolvido e utilizado com sucesso no trabalho de Esposito (2010). O autor utilizou medidas da vazão mássica $(\mathrm{g} / \mathrm{s})$ para calcular o balanço de energia da camisa no trabalho realizado sobre calorimetria de balanço de calor. Medidas da vazão volumétrica $(\mathrm{L} / \mathrm{min})$ foram mostradas em tempo real por meio de um sistema supervisório. O diferencial do presente trabalho é a redução do período de amostragem, tempo entre a obtenção de uma media e outra, que passou de $10 \mathrm{~s}$ para $2 \mathrm{~s}$. Esta alteração exigiu uma reformulação completa do algoritmo utilizado originalmente. Além disso, o método foi aplicado em uma nova unidade experimental. Novas características de operação foram identificadas e incorporadas para que as medições se tornassem ainda mais robustas e confiáveis.

\section{METODOLOGIA}

O medidor, o hidrômetro Unimag, é construído com uma turbina que se movimenta de acordo com o escoamento do fluido. Um acoplamento magnético, que isola as partes móveis (turbina) do restante do medidor, detecta a passagem das hastes da turbina em um determinado ponto. Isso gera um sinal que pode ser recebido por outros sensores. Nesta aplicação, o sensor Cyble foi acoplado ao medidor Unimag, para se interpretar os pulsos advindos do medidor de volume e envio de sinal à placa de aquisição de dados. 
Utilizando o Cyble V2 com KLF $=1$ (LF (low frequency) $=\mathrm{HF}$ (high frequency) multiplicado pelo fator K), a cada litro de fluido escoado é emitido um ciclo de pulso para o sistema de aquisição de dados.

A Figura 1 mostra o medidor Unimag, o acoplamento do Cyble ao medidor e a identificação dos condutores do sensor. Todos os condutores de sinal (branco, verde, amarelo e vermelho) possuem tensão positiva em relação à referência (condutor preto). Os condutores de sinal devem ter sua corrente limitada com o uso de um resistor; para este caso foi utilizado um resistor de $100 \mathrm{ohm}$.

Figura 1 - Medidor Unimag, sensor Cyble e ligação do sensor.
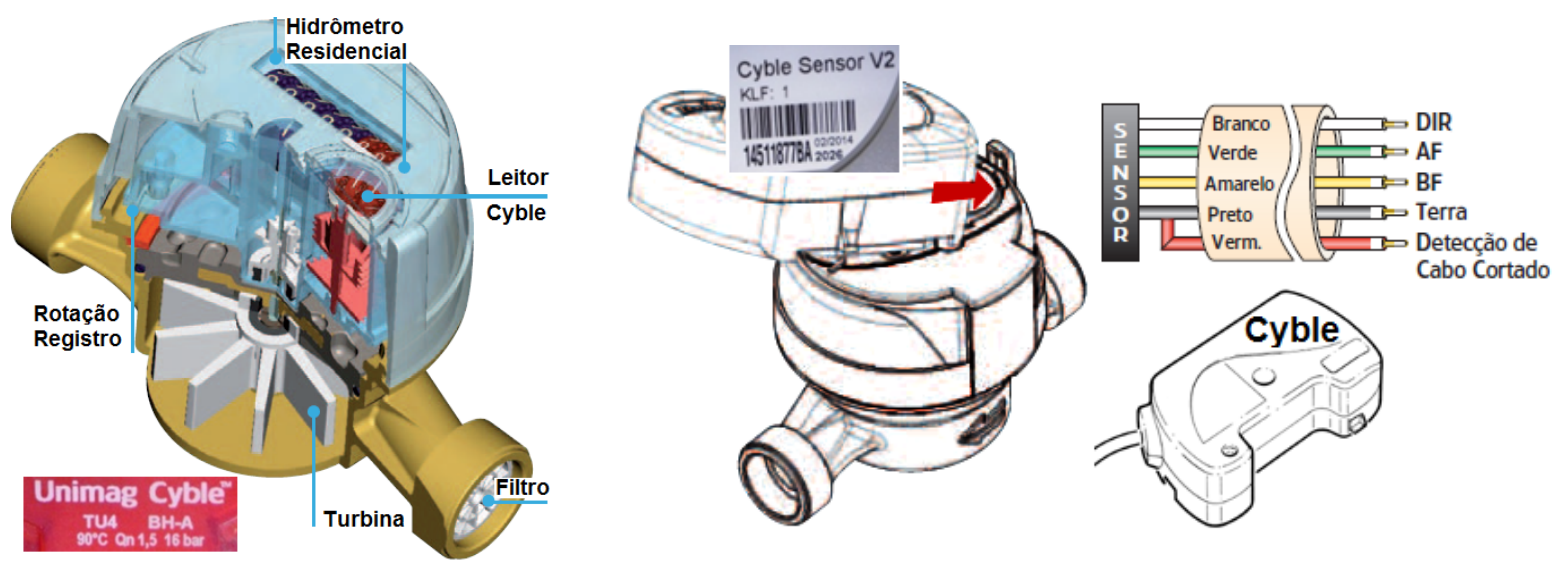

A Figura 2 mostra parte do código utilizado no tratamento dos dados. Nesta figura pode-se observar como o vetor VetDescida foi declarado e sua função na identificação de mudanças de estado, aqui chamadas de transição.

Figura 2 - Código no software Matlab

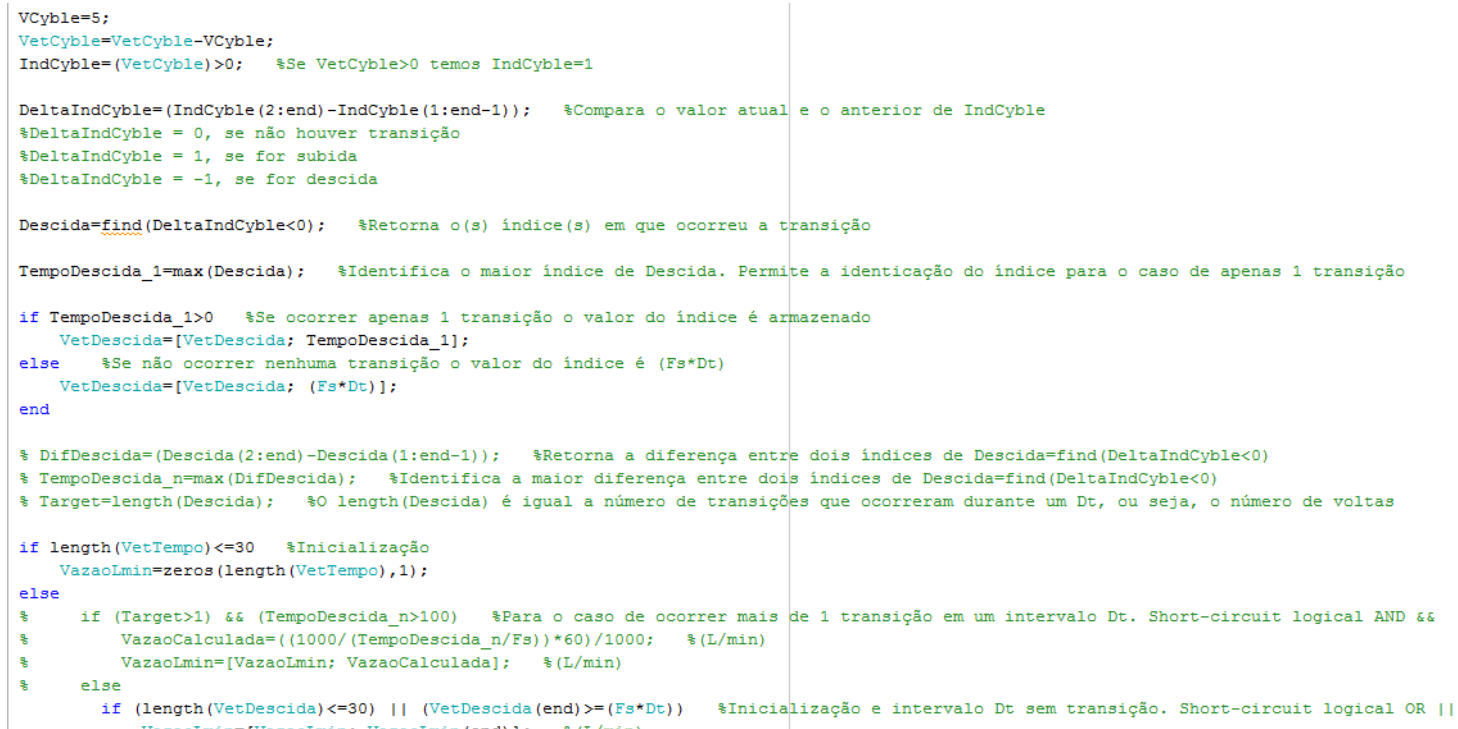


A partir dos índices das bordas de descida de cada ciclo, a vazão foi calculada segundo a Equação 1.

$$
V(L / \text { min })=\frac{F s * 60}{((F s * D t)-V e t D e s c i d a(\text { end }-3))+V e t D e s c i d a(\text { end }-2)+V e t D e s c i d a(\text { end }-1)+\operatorname{VetDescida}(\text { end })}
$$

onde, $V$ é a vazão, $F s$ é a frequência, $D t$ é o período de amostragem.

A Tabela 1 apresenta as instruções utilizadas na confecção do algoritmo para a medição da vazão. Cada período de amostragem de $2 \mathrm{~s}$ correspondeu a 2000 amostras e foram necessários $60 \mathrm{~s}$ ou 30 períodos de amostragem, para a inicialização e obtenção do valor correto da grandeza medida. Após a inicialização, o valor vazão foi atualizado a cada $2 \mathrm{~s}$.

Tabela 1 - Instruções utilizadas no algoritmo de medição da vazão.

\begin{tabular}{lcc}
\hline $\begin{array}{c}\text { Número de } \\
\text { períodos }\end{array}$ & VetDescida: com $F s=1000 H z$ e período de amostragem, $D t=2 s$. & $\begin{array}{c}\text { Vazão } \\
L / \text { min }\end{array}$ \\
\hline $4 \leftrightarrow 4,2 s$ & $1999+2000+2000+1$ & 14,99 \\
$4 \leftrightarrow 8,0 s$ & $1+2000+2000+1999$ & 7,50 \\
$5 \leftrightarrow 10,0 s$ & $1+2000+2000+2000+1999$ & 6,00 \\
$6 \leftrightarrow 12,0 s$ & $1+2000+2000+2000+2000+1999$ & 5,00 \\
$7 \leftrightarrow 14 s$ & $1+2000+2000+2000+2000+2000+1999$ & 4,29 \\
$8 \leftrightarrow 16 s$ & $1+2000+2000+2000+2000+2000+2000+1999$ & 3,75 \\
$9 \leftrightarrow 18 s$ & $1+2000+2000+2000+2000+2000+2000+2000+1999$ & 3,33 \\
$10 \leftrightarrow 20 s$ & $1+2000+2000+2000+2000+2000+2000+2000+2000+1999$ & 3,00 \\
\hline
\end{tabular}

\section{RESULTADOS E DISCUSSÃO}

Na Figura 3 são apresentados os dados obtidos diretamente a partir da leitura do sensor Cyble, mais especificamente, os dados que compõem o vetor VetCyble do algoritmo de programação. A Figura 3(a) apresenta as características de tensão do pulso transmitido pelo sensor: a borda de descida ocorre na amostra 366 e a borda de subida na amostra 432. Como a taxa de amostragem é de $1 \mathrm{kHz}$, o tempo gasto pelo ciclo de pulso é $66 \mathrm{~ms}$. Na Figura 3(b) observa-se o posicionamento temporal dos pulsos emitidos pelo sensor. A primeira borda de descida ocorre na amostra 366 e a segunda ocorre na amostra 6521. Para a determinação da vazão, em litros por minuto, foi necessário determinar o tempo entre um pulso e outro. A informação transmitida é de que, neste intervalo, o volume de 1 litro de fluido passou pelo hidrômetro. Há uma diferença de 6155 amostras entre os dois pulsos, o que caracteriza um tempo de 6,155 s e corresponde a uma vazão de 9,8 L/min para este experimento. Esta é a vazão máxima que pode ser obtida com a motobomba utilizada.

Figura 3 - Dados obtidos pelo sensor Cyble. 


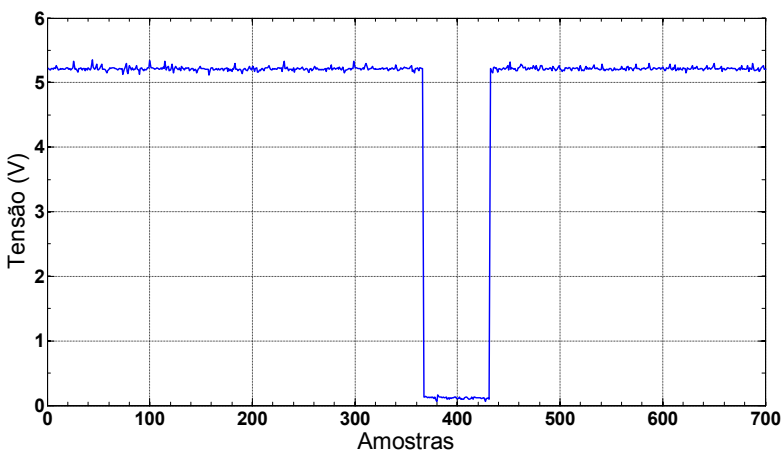

(a)

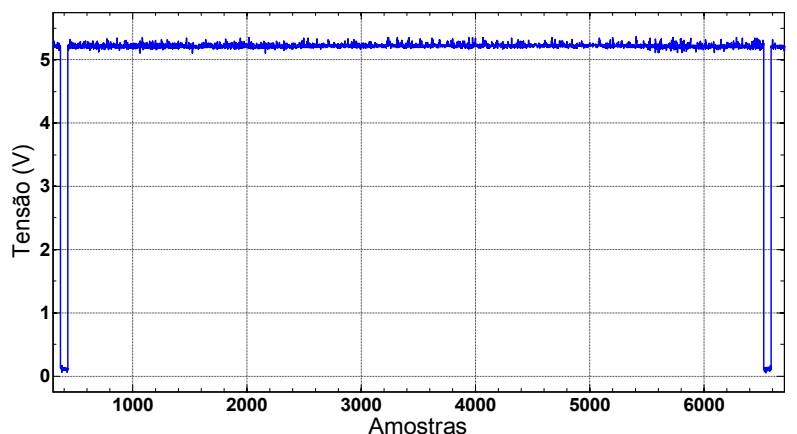

(b)

O valor médio de operação do Cyble, em volts, é de 5,2 V para nível lógico alto e $0,06 \mathrm{~V}$ para nível lógico baixo. No Matlab, para facilitar a identificação dos índices onde ocorrem os pulsos, foi subtraído do ponto de operação o valor de $V C y b l e=5 \mathrm{~V}$. A Figura 4 mostra o comportamento dinâmico da variável IndCyble, que indica o nível lógico do sinal do sensor.

Figura 4 - Nível lógico do sinal do sensor Cyble.

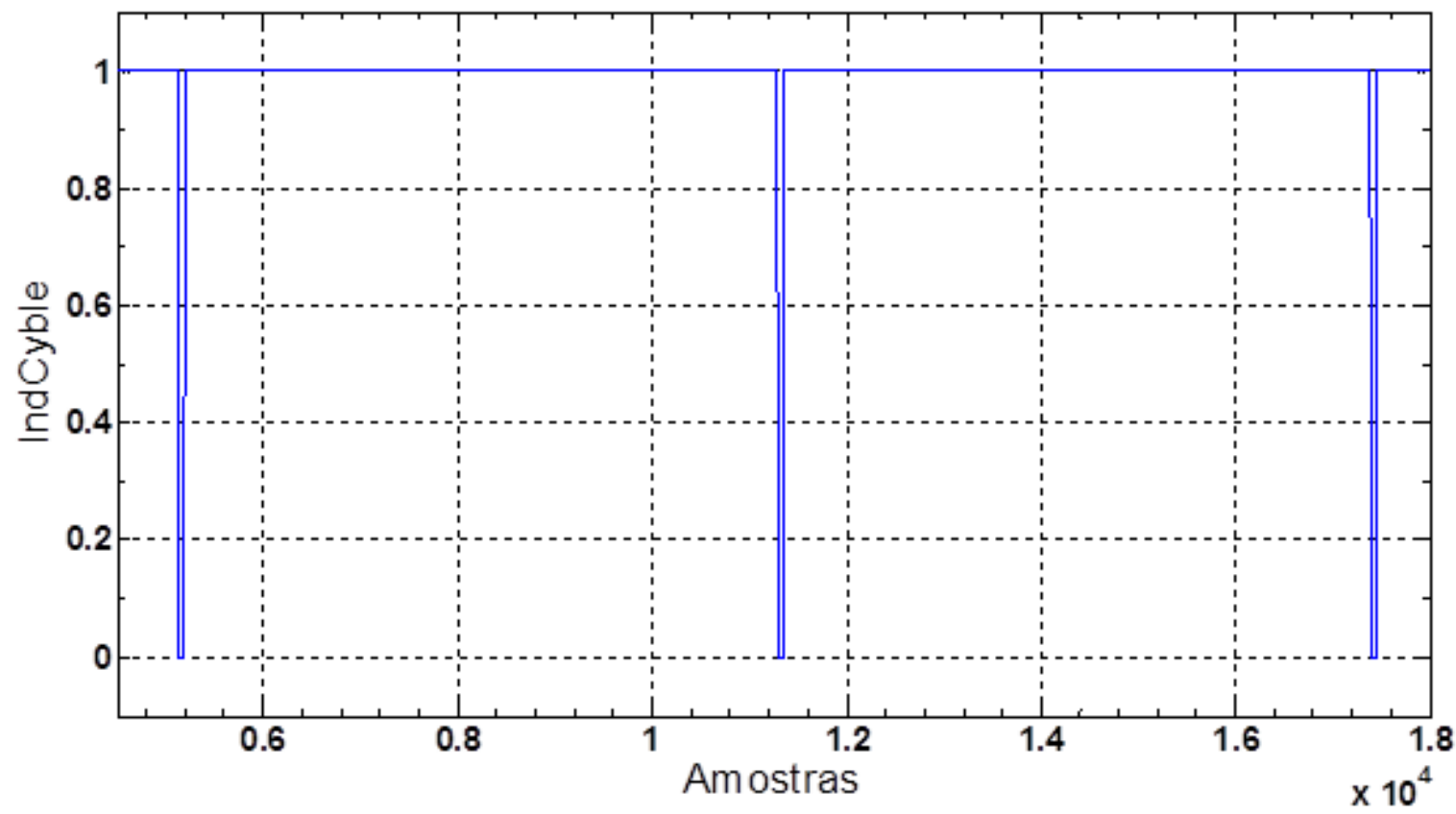

É necessário considerar o período de amostragem para que o valor da vazão seja atualizado corretamente na tela do sistema supervisório. Cada período de amostragem $(2 \mathrm{~s})$ foi classificado com o uso da variável DeltaIndCyble, o qual é gerado comparando-se o valor atual com o anterior do sinal do sensor (Figura 5). Se não houver transição, o índice é 0; se houver uma borda de subida, o índice é 1; e caso houver uma borda de descida, o índice é -1.

Figura 5 - Classificação dos períodos de amostragem. 


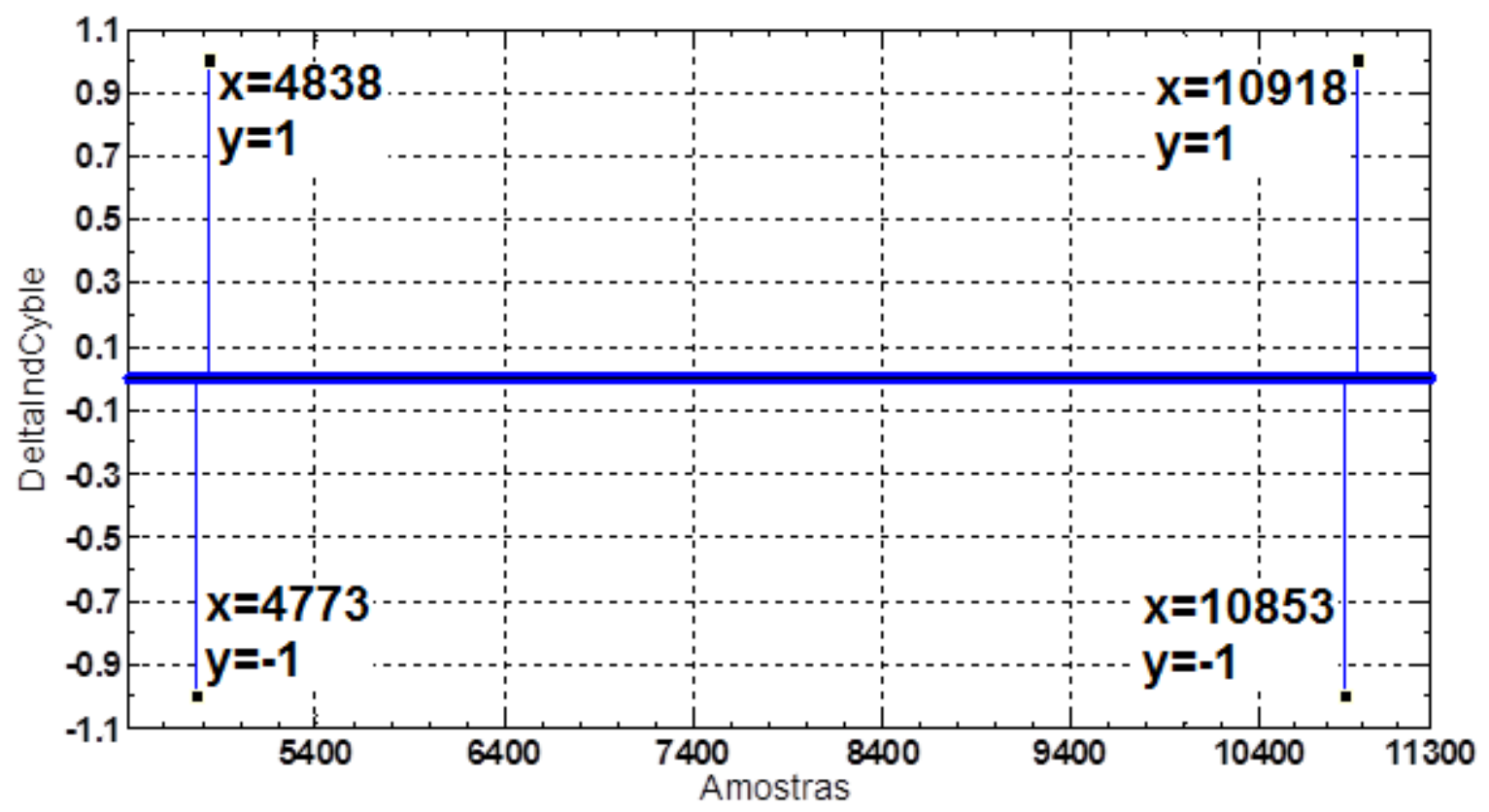

O comando find do Matlab foi utilizado para localizar todos os elementos em que ocorreram transições de descida. Este comando pode localizar índices que não correspondem a transições verdadeiras $(1 \mathrm{~L})$. Por este motivo, optou-se por considerar apenas a maior distância entre dois índices, utilizando-se o comando max (linha 16 do código apresentado na Figura 2). Este comando identifica o maior índice, já que experimentalmente é impossível a ocorrência de mais de uma transição em um período de amostragem. Caso ocorra transição, o valor do índice é armazenado; caso contrário, é armazenado o número total de amostras analisadas visto que a frequência de amostragem é $1 \mathrm{kHz}$ e o período de amostragem é $2 \mathrm{~s}$.

A Figura 6 mostra os dados de medição da vazão. Observa-se a amplitude das medições ao se atuar com uma válvula em patamares. Com o auxílio de um medidor de pressão, também ligado na rede hidráulica em circuito fechado com o banho ultra termostático, identificou-se a coerência das medições. Por exemplo, pressão 0 bar (motobomba desligada) corresponde à uma vazão igual a zero, e 0,8 bar (motobomba ligada) à vazão máxima. As oscilações de vazão foram instantaneamente observadas no comportamento dinâmico da pressão.

Figura 6 - Variação da vazão do fluido de arrefecimento. 


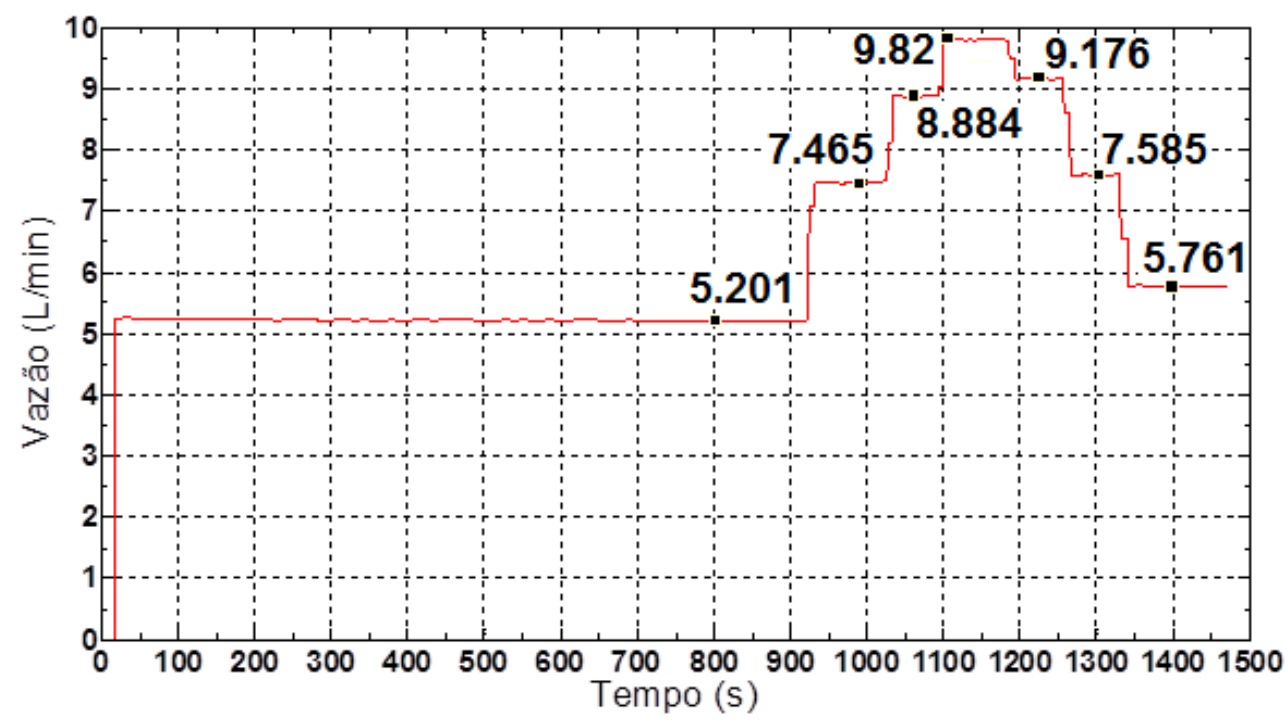

\section{CONCLUSÕES}

A utilização deste método demonstrou-se confiável, uma vez que o valor da vazão foi obtido corretamente ao final de cada ciclo de tratamento dos dados. Apesar do valor da vazão ser atualizado apenas a cada $2 \mathrm{~s}$, ao final de cada período de amostragem, para o processo em questão, este intervalo é satisfatório, considerando-se que a constante de tempo do processo é da ordem de minutos. Em estudos anteriores do grupo, análises calorimétricas foram realizadas em tempo real, com atualizações do calor gerado pela reação a cada $10 \mathrm{~s}$, ou seja, o baixo custo e a eficiência deste sistema empregado para a medição da corrente quente (até $90{ }^{\circ} \mathrm{C}$ ) pode ser considerada uma ótima opção para o uso em aplicações onde se deseja versatilidade e domínio sobre o sistema de medição.

\section{REFERENCIAS}

BERGMAN, G. Avaliação metrológica de sistemas de medição de vazão. 2011. 95 f. Trabalho de Conclusão de Curso (Graduação) - Curso de Engenharia de Controle e Automação, Centro de Ciências Exatas e Tecnológicas, Univates, Lajeado.

ESPOSITO, M., Instrumentação e monitoramento em tempo real de reatores de polimerização através de técnicas calorimétricas. 2010. 155f. Tese (Doutorado em Engenharia Química). Universidade Federal de Santa Catarina, Florianópolis.

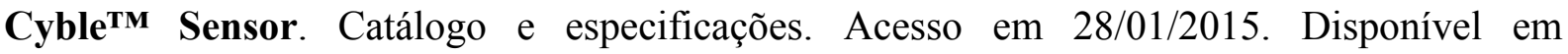
$<$ https://www.energasequipamentos.com.br/manuais/catalogos/Cyble_Sensor_pb_PT_lam_07 $-13 . \mathrm{pdf}>$.

UnimagTM Medidor. Catálogo e especificações. Acesso em 28/01/2015. Disponível em $<$ https://www.energasequipamentos.com.br/manuais/catalogos/Unimag\%20Agua\%20Quente PT.pdf>. 